\title{
Indicadores e instrumentos de obtención de datos del Observatorio Urbano Arquitectónico de Entornos Saludables OUAES
}

\author{
Indicators and instruments for obtaining data from the \\ Urban Architectural Observatory of Healthy Enviroments OUAES
}

\author{
Irma Elisa Palacios ${ }^{\text {a }}$ \\ Erika Benitez ${ }^{b}$ \\ Daniel Alejandro Gómez ${ }^{\mathrm{c}}$ \\ José René Morgado ${ }^{d}$
}

Recibido: 11 de enero de 2021.

Aceptado: 05 de abril de 2021.

RESUMEN: El artículo tiene como finalidad presentar los 38 indicadores del Observatorio Urbano Arquitectónico de entornos saludables y los 9 instrumentos de obtención de datos para los indicadores extensivos. Se mostrará como el OUAES estructura y clasifica esos 38 indicadores en 3 categorías las cuales están organizadas en indicadores básicos (IB), indicadores clave (IC) e indicadores extensivos (IE). La obtención de los datos para el cálculo de algunos indicadores se

\footnotetext{
a Observatorio Urbano Arquitectónico de Entornos Saludables (OUAES), Facultad de Arquitectura, región Córdoba, Universidad Veracruzana, México. Contacto: irpalacios@uv.mx*Autora para correspondencia

b Observatorio Urbano Arquitectónico de Entornos Saludables (OUAES), Facultad de Arquitectura, región Córdoba, Universidad Veracruzana, México. Correo: ebenitez@uv.mx

c Observatorio Urbano Arquitectónico de Entornos Saludables (OUAES), Facultad de Arquitectura, región Córdoba, Universidad Veracruzana, México. Correo: dangomez@uv.mx

d Observatorio Urbano Arquitectónico de Entornos Saludables (OUAES), Facultad de Arquitectura, región Córdoba, Universidad Veracruzana, México. Correo: rmorgado@uv.mx
} 
recaba mediante instrumentos diseñados por encuestas de las cuales los resultados son datos estadísticos derivados y básicos.

Palabras clave: Indicadores, instrumentos de medición, indicadores básicos, indicadores clave, indicadores extensivos.

ABSTRACT: This paper aims to present the 38 indicators of the Urban Architectural Observatory of healthy environments and the 9 data collection instruments for the extensive indicators. It will be shown how the OUAES organizes and classifies these 38 indicators into 3 categories which are organized into basic indicators (IB), key indicators (CI) and extensive indicators (IE). Obtaining the data for the calculation of some indicators is collected through instruments designed by surveys of which the results are derived and basic statistical data.

Keywords: Indicators, measurement instruments, basic indicators, key indicators, extensive indicators.

\section{Introducción}

$\mathrm{E}$ s importante considerar que al monitorear y observar el fenómeno que producen las ciudades no saludables, aspecto relacionado al proceso de resiliencia urbana y arquitectónica, es relevante considerar los factores que inciden en la salud relacionada con los entornos públicos y privados en donde la comunidad -niños, adolescentes, adultos y tercera edad- desarrollan todas sus actividades.

Un indicador es un instrumento para clarificar y definir objetivos de forma precisa, ya que son medidas verificables de cambio o resultado diseñados para contar con un estándar contra el cual evaluar, estimar o demostrar el progreso con respecto a metas establecidas para alcanzar los objetivos; así lo refiere SNIEG (2020). Por lo anterior, el OUAES realiza el monitoreo permanente y de manera periódica de los 38 indicadores que se muestran a lo largo del artículo. Se debe considerar y tener presente que el monitoreo es un proceso continuo y sistemático de recopilación y análisis de datos SNIEG (2020). Por esa razón en el observatorio se trabajan los instrumentos de obtención de datos e indicadores extensivos de manera sistemática, organizada en reportes semestrales.

El monitoreo que se realiza tiene indicadores y temáticas asociadas con entornos saludables y con la percepción espacial de manera urbana y arquitectónica; en ese sentido, monitorea, observa, mide y registra indicadores de salud integral, percepción espacial, entornos saludables, esperanza de vida, percepción de bienestar y huella ecológica. Las temáticas asociadas con las que relaciona la observación son: espacio público, vivienda, infraestructura y servicios, regeneración y planeación urbano, bioclimatismo, vulnerabilidad y factores de riesgo, movilidad, sustentabilidad, patrimonio histórico y peritaje urbano. Con estos datos y el registro, el OUAES genera insumos para la generación y la aplicación del conocimiento en la investigación, tales como: información estadística básica y derivada, información cartográfica, infografías, catálogos georreferenciados, reportes 
semestrales, reportes especiales, catálogo de problemáticas urbanas y arquitectónicas, metodologías y encuestas.

\section{Sistemas de indicadores del OUAES}

Las constantes del fenómeno observado en el OUAES están definidas por los siguientes indicadores: Salud integral, percepción espacial, ambientes saludables y los 3 componentes para identificarlos, que son la esperanza de vida, la percepción de bienestar y la huella ecológica. Las variables que inciden en el análisis y monitoreo del fenómeno son: el tiempo de permanencia en los espacios y las características urbanas contextuales.

Los indicadores que se consideran en materia de salud y elementos que inciden en el monitoreo de las ciudades respecto a ser o no saludables son:

- Indicadores indirectos, vinculados con la población rural y urbana; e indicadores directos vinculados con servicios y con infraestructura.

- Indicadores a nivel internacional, directamente ligados a los de la OMS, OPS, OCDE, Sustainable Development Report 2019, SDG Index and Dashboard Report 2017, ODS 2030, ONU.

- Indicadores a nivel nacional que utiliza el observatorio: INEGI, SS, INSP, IMSS, CONEVAL, SMAES, Índice de ciudades sostenibles de Citibanamex.

Los instrumentos de análisis que el OUAES, utiliza para la generación de la información estadística está basada en estadística básica y derivada. En el caso de la aplicación de la estadística básica refiere al diseño de encuestas específicas para el fenómeno y el levantamiento de información por muestreo. Considerando como unidad de muestreo el AGEB.

En el caso de la aplicación de la estadística derivada se utilizan los registros estadísticos y bancos de datos de fuentes confiables nacionales e internacionales. El OUAES basa sus instrumentos en la metodología de INEGI y Sustainable Development Report 2019, SDG Index and Dashboard Report 2017.

El observatorio utiliza fuentes de información para la correlación e interpretación de datos; considerando en la observación del fenómeno de estudio las siguientes encuestas: ENVIPE 2017, ENCIG 2017, MOPRADEF, MOLEC, MODUCULT y MOPRADEF; ya que se identifican datos relevantes asociados al fenómeno de observación y monitoreo.

\section{Batería y clasificación de Indicadores del Observatorio Urbano Arquitectónico de Entornos Saludables OUAES}

El OUAES, en relación con la metodología que plantea, tiene el objetivo de recopilar, monitorear, analizar y generar información estadística vinculada con la observación del fenómeno que producen las ciudades no saludables relacionada al proceso de resiliencia urbana y arquitectónica, compartiendo conocimiento especializado para entender las ciudades como sistemas sociales y urbanos que inciden directamente de la salud y bienestar de los habitantes. Por lo cual observa 38 
indicadores. Los cuales, de acuerdo con su categoría, característica de la fuente de información y tipo de indicador (cualitativo o cuantitativo) se clasifican de la siguiente manera: la categoría A son 11 Indicadores básicos (IB), se obtienen de la estadística derivada; la categoría B, 9 Indicadores Clave principales (IC) y se obtienen de la estadística básica; y la categoría C, incluye 18 Indicadores Extensivos (IE) que se generan con estadística derivada e instrumentos diseñados y calibrados por el OUAES para poder recopilar la información.

Los 11 Indicadores Básicos (IB), correspondientes a la categoría A, se monitorean y observan con una matriz check list en donde la finalidad es identificar en la ZMC y ZMO las características principales que determinan una ciudad saludable. La matriz check list para identificar estas características el OUAES se basa en Latapi (2014).

Estos 11 Indicadores Básicos (IB) son:

1. Ambiente físico de alta calidad, limpio y seguro.

2. Calidad en la vivienda.

3. Ecosistema estable y sostenible a largo plazo.

4. Comunidad cohesionada y no explotable.

5. Alto grado de participación y control de los ciudadanos sobre las decisiones que afectan su vida, salud y bienestar.

6. Satisfacción de las necesidades básicas de alimento, agua, vivienda, ingresos, seguridad y trabajo para todos los habitantes.

7. Acceso a la variedad de experiencias y recursos que faciliten el contacto, interacción y comunicación de sus habitantes.

8. Economía diversa, vital e innovadora.

9. Fomento de la conexión con las culturas del pasado, herencia biológica y con otros grupos e individuos.

10. Acceso para todos a los servicios de salud.

11. Elevado estado de salud y bajos niveles de enfermedad.

Los que denominamos Indicadores clave principales (IC) de la categoría B son los que otorgan la referencia o marco teórico para poder generar los cálculos estadísticos correlacionados del OUAES. Estos indicadores claves refieren fórmula específica e identificada por organismos como: INEGI, Happy planet index, CONEVAL, OMS, ONU.

Los Indicadores Clave principales (IC) son 9 y son los siguientes:

12. Índice de rezago social.

13. Índice de huella ecológica.

14. Índice de esperanza de vida.

15. Índice de percepción de bienestar.

16. Índice de felicidad según la fuente directa de Happy planet index la cual se mide con tres componentes: la esperanza de vida, la percepción de bienestar y la huella ecológica.

17. Indicadores indirectos vinculados con la población urbana y rural.

18. Indicadores directos vinculados con servicios y con infraestructura. 
19. Factores de riesgos a la salud en espacios abiertos y cerrados.

20. Indicadores referidos en la carta de Ottawa (1986) indispensables para la salud: paz, techo, educación, comida, ingreso, ecosistema estable, recursos sostenibles, justicia social y equidad.

Respecto a los Indicadores Extensivos (IE), correspondientes a la categoría C, se obtienen y generan por medio de encuestas diseñadas con datos estadísticos derivados y básicos. En el caso de estos indicadores el OUAES a la fecha tiene diseñados 2 encuestas semestrales; una de ellas la denominada ESES-EES se usa para la observación a escala arquitectónica y la ESES-EURB para la escala urbana. Se cuenta así también con 8 encuestas diseñadas para la obtención y medición de los indicadores extensivos (IE).

Los indicadores extensivos (IE) de la categoría C son:

21. Servicios básicos

22. Infraestructura

23. Estructura vial

24. Mobiliario urbano

25. Espacios públicos

26. Vegetación y áreas verdes

27. Accesibilidad urbana

28. Percepción espacial

29. Legibilidad urbana

30. Sistemas de acceso

31. Territorio

32. Patrimonio histórico

33. Vulnerabilidad espacial

34. Equipamiento urbano

35. Vivienda

36. Factores ambientales y entorno

37. Estilo de vida

38. Factores biológicos

Los indicadores extensivos sirven como una base de datos para poder generar los cálculos estadísticos de correlación de variables y constantes para la obtención de porcentaje requeridos para la medición de los entornos saludables. El cálculo se realizará con la unidad mínima espacial definida como AGEB. Los cuales son indicadores directos vinculados con servicios e infraestructura.

Los instrumentos de medición para obtener los indicadores extensivos se les asigna una valoración considerando la siguiente escala y los criterios de valoración según el indicador a evaluar. Esta valoración se hace con relación a la zona en general y al check list de los principales servicios e infraestructura existente básica. En los que se consideran 5 parámetros:

- Excelente: la zona cuenta con el espacio o servicio en perfectas condiciones, no hay escasez de los elementos indicados y existe la cantidad necesaria para la zona. Rangos de valoración estimativa (5) del $80 \%$ al $100 \%$. 
- Bueno: La zona cuenta con el espacio o servicio en condiciones aceptables y la cantidad justa suficiente. Rango de valoración estimativa (4) del 60 al $79.99 \%$.

- Regular: La zona casi no cuenta con el espacio o servicio, hace falta más y de los que hay su condición es deficiente. Rango de valoración estimativa (3) del 40 \% al 59.99 \%.

- Malo: La zona cuenta con el espacio mínimo o servicios necesarios, están en pésimas condiciones. Rango de valoración estimativa (2) del $20 \%$ al 39.99\%.

- Deficiente: La zona no tiene ninguno de los espacios necesarios. Rango de valoración estimativa (1) del $0 \%$ al $19.99 \%$.

Los indicadores relacionados a la accesibilidad urbana, percepción del espacio e imagen urbana, se evaluaron de la siguiente manera, utilizando la escala de valoración sobre los rangos estimativos de: 5 del $80 \%$ al $100 \%$, 4 del 60 al $79.99 \%$, 3 del $40 \%$ al $59.99 \%$, 2 del $20 \%$ al 39.99 \%, 1 del $0 \%$ al $19.99 \%$. Considerando el 5 el más alto y el 1 el más bajo rango.

Referente a las encuestadas diseñadas por el OUAES para la obtención de datos se muestra el listado de las 9 encuestas para obtención de los Indicadores Extensivos (IE).

1. EES-A-F1/01 Encuesta de entornos saludables. Paxtian (2019).

2. FRDA-F2/02 Ficha de registro y levantamiento de alteraciones y deterioros por nodo y por larguillo (por recorridos) con uso de tecnología aplicada. Instrumento de recopilación de datos Drone Mavic Pro. Paxtian (2019)

3. DZE-FC/03 Ficha de delimitación de la zona de estudio y contextualización.

4. IMEPAV /04 Ficha instrumento para valorar los espacios públicos, jardines, áreas verdes. Ficha de percepción fenomenológica del espacio público.

5. CEGH-IC/05 Ficha instrumento diseñado para la obtención de datos ingenieriles denominada: Instrumento construcción estructuras geotecnia e hidráulica. Hernández \& Reyes (2020).

6. EPSR-OU/06 Ficha instrumento que se denomina Encuesta de la percepción de la satisfacción residencial.

7. EEP-ES/07 Encuesta espacios públicos relacionada a sus diferentes categorías y diagnostico en relación con espacios saludables. Identifica y categoriza los espacios públicos de acuerdo con su nivel de elementos que alteran el espacio e identifica el porcentaje de espacio saludable.

8. Encuesta especial COVID-19 denominada: "Percepción espacial para entornos saludables en espacio público de educación superior. OUAES (2020)”. Es una encuesta diseñada para generar datos que sirvan para aplicar la metodología de reconversión espacial de espacios públicos de educación superior a la nueva normalidad. Con la finalidad de organizar espacialmente el partido arquitectónico y urbano de las edificaciones para que sean saludables a todos los usuarios. La encuesta se encuentra organizada en 7 apartados: a) Generales del Covid-19, b) Tipos de usuarios, c) Riesgos generales, d) Percepción del regreso a la nueva normalidad, e) Diagnostico de zonas de contagio y alto riesgo, F) Lineamientos del regreso a la nueva normalidad y g) Espacios saludables OUAES. Se puede consultar en: https://www.uv.mx/orizaba/ouaes/reporte-especial-covid-19/

9. Encuesta para generar el diseño urbano arquitectónico de ambientes universitarios saludables. Esta encuesta fue diseñada desde el 2018 con el objetivo de generar datos 
específicos en la observación de espacios no saludables OUAES (2018). La estructura general de la encuesta y resultados se pueden consultar en:

Revista No.6 (2018) https://uvserva.uv.mx/index.php/Uvserva/article/view/2585

\section{Conclusiones}

Los indicadores y datos que el OUAES produce y genera nos sirve para contribuir a la sociedad de la aplicación y generación del conocimiento a nivel regional, nacional e internacional; que se pone a disposición de toda la comunidad universitaria: estudiantes, docentes, investigadores, administrativos, colaboración académica con organismos especializados. El observar y medir con indicadores genera eficiencia, proceso y satisfacción en la objetividad por consenso atinado y pertinente de trayectorias adecuadas en las propuestas de soluciones a problemas identificados y focalizados. Toda la información sirve a instituciones y organismos especializados. Aportando datos confiables para poder generar espacios urbanos y arquitectónicos que ofrezcan al ser humano: bienestar, seguridad, conectividad, accesibilidad universal, servicios básicos, proporción y escala; todos estos elementos convergentes en la observación del fenómeno respecto a la resiliencia en la percepción espacial urbana y arquitectónica.

Para el OUAES es indispensable generar información sistematizada y confiable para el desarrollo de estrategias, toma de decisiones, procesos de planeación, fomento a la cultura de la estadística, conciencia de la responsabilidad social universitaria que aporte a las agendas de problemas, fomento y fortalecimiento de la investigación desde los cuerpos académicos. Aportando y contribuyendo directamente a la: RSU, sustentabilidad, a los ODS 2030, a la sociedad y a toda la comunidad universitaria regional, nacional e internacional.

\section{Referencias}

SNIEG. (2020). Glosario del Sistema nacional de información estadística y geográfica. https://snieg.mx/DocumentacionPortal/Normatividad/vigente/Glosario_Normativi dad_SNIEG.pdf

Latapí, J. (2014). Hacia un entorno Saludable en las comunidades mexicanas. Conacyt. https://www.anmm.org.mx/publicaciones/CAnivANM150/L17-Hacia-un-entornosaludable-en-las-cominidades-mexicanas.pdf

Paxtian, M.L. (2019) Tesis en colaboración con Cuerpo académico UV-CA-498 y OUAES. Estrategia metodológica para generar agenda de datos urbanos y arquitectónicos del Observatorio Urbano Arquitectónico de Entornos Saludables. Estrategias tecnológicas. Caso de estudio: Ciudad de Córdoba. Universidad Veracruzana. México. Facultad de Arquitectura Córdoba.

Hernández, 0., Reyes, Q. (2020). Tesis en colaboración con Cuerpo académico UV-CA-498 y OUAES. Colaboración en la Investigación metodológica para generación de datos de ingeniería civil para Observatorio Urbano Arquitectónico de Entornos Saludables en la zona metropolitana de Orizaba, Ver. zona 9, zona 34 y zona 20. Universidad Veracruzana. Facultad de ingeniería Civil. México. Campus Ixtaczoquitlán, Ver. 
OUAES. (2020). Universidad Veracruzana. https://www.uv.mx/orizaba/ouaes/reporteespecial-covid-19/

OUAES. (2018). Metodología aplicada: una estrategia de diseño para generar ambientes universitarios saludables, UVserva, (6), 84-92

https://doi.org/10.25009/uvserva.v0i6.2585 